\title{
The therapeutic alliance in psychotherapy for adolescent depression: differences between treatment types and change over time
}

\begin{abstract}
:
Although the alliance is usually considered a generic factor common to all therapies, there are theoretical reasons to suspect that the alliance may develop differently in different types of therapies. Yet, in youth psychotherapy little is known about this issue to date. This study investigated whether the mean strength of the alliance, as well as its trajectory over time, differed between three equally effective psychological treatments for adolescent depression. Data were drawn from the *anonymised* study, a randomized controlled trial comparing cognitive-behavioural therapy (CBT) and short-term psychoanalytic psychotherapy (STPP) versus a brief psychosocial intervention (BPI) in the treatment of adolescent depression. Adolescents' $(\mathrm{N}=338)$ and therapists' $(\mathrm{n}=159)$ ratings of the alliance were collected using the Working Alliance Inventory short form (WAI-S) at 6, 12 and 36 weeks after randomization. Data were analysed using multilevel linear models. Results showed that adolescents' and therapists' mean alliance ratings differed between treatment types, being highest in CBT, and lowest in the STPP at all time points. Mean therapists' alliance ratings increased slightly over time in all arms, while mean adolescents' ratings were stable over time in CBT and BPI, but slightly increased in the STPP group. These findings suggest that the mean strength of the alliance differs between treatment type and future research is required to help pinpoint what factors contribute to these differences and their relationship with treatment outcomes.
\end{abstract}

Keywords: Alliance; Youth Psychotherapy; Working-Alliance-Inventory; CognitiveBehavioural Therapy, Psychoanalytic Psychotherapy

The therapeutic alliance refers to the relationship between patient and therapist in terms of their ability to collaborate in the therapeutic process (Bordin, 1979). One of the main 
attractions of the concept is the fact that it is considered a "common factor" (Horvath, 2018). Common factors refer to generic psychotherapy variables which are assumed to be present and play an important role in all types of therapy (Zilcha-Mano et al., 2019). By contrast, specific factors refer to therapeutic techniques that are particular to a psychotherapeutic orientation (e.g., cognitive restructuring in cognitive therapies or transference interpretations in psychodynamic therapies) (Zilcha-Mano et al., 2019). Concerning the relationship between patient and therapist, the alliance is one of the most investigated common factors in psychotherapy (Horvath, 2018).

There is a growing body of research showing that good alliance is associated with favourable outcomes across treatments in youth psychotherapy (Karver et al., 2018). These findings appear to support the assumption that a good alliance is a 'common' ingredient of efficacious therapies (Horvath, 2018). However, differently from the adult alliance literature, the strength of the alliance-outcome association in youth psychotherapy was found to differ across treatment types, with stronger associations in behavioural versus nonbehavioral therapies (Shirk et al., 2011; Karver et al., 2018). Even if the alliance is considered important in all types of therapy, it might not necessarily operate in the same way across different types of treatment. Yet, to date little attention has been paid to understanding whether treatment types influence the alliance strength. To better understand the role of the alliance we need to learn more about what influences its development and treatment type might be an important factor to consider when assessing the alliance with young people. Hence, determining whether certain treatments facilitate or hinder the development of an alliance represents an important research goal.

From a theoretical perspective, even Bordin (1979), who developed the most widely used 'pan-theoretical' description of the alliance, acknowledged that its characteristics might differ across therapies. He described the alliance as the agreement between patient and therapist 
on therapy goals and tasks in the context of an emotional bond and clarified that the degree to which each therapy relies on these different alliance dimensions might differ (Bordin, 1979). Several authors have supported this idea (Hatcher, 2010; Hatcher \& Gillaspy, 2006; Webb et al., 2011). For example, it has been argued that cognitive-behavioural therapies (CBT) might place more emphasis on collaboration on tasks and goals (Bordin, 1979; Castonguay et al., 2006; Muran \& Barber, 2010; Raue \& Goldfried, 1994; Webb et al., 2011) compared to psychodynamic and humanistic therapies, which might focus more on the bond, i.e. the emotional connection between therapist and patient (Bordin, 1979; Freud, 1946; Muran \& Barber, 2010; Webb et al., 2011). However, these hypotheses have not been empirically tested, especially in youth psychotherapy (DeRubeis et al., 2005; Ulvenes et al., 2012).

The majority of youth alliance research has investigated the relationship between alliance and outcome, and little is known about whether certain treatments achieve better alliance than others. The few studies reporting on the differences in mean alliance ratings across treatment types have yielded mixed results. An RCT, examining the alliance-outcome link in manualised trauma-focused CBT versus therapy as usual for traumatised youths, found no evidence that that youth-rated mean alliance differed between treatments (Ormhaug et al., 2014). Langer and colleagues (2011) presented evidence that early in treatment observational ratings of the alliance were higher in manual-guided CBT than in non-manualized treatment delivered in community-based service settings. However, the two groups converged over time and there was no evidence that both observer- and youth-reported alliance differed by treatment conditions (Langer et al., 2011). In contrast, Hogue and colleagues (2006) found that early observational ratings of the alliance were significantly higher in family therapy than in CBT for adolescents with substance abuse issues (Hogue et al., 2006). Furthermore, McLeod and colleagues (2016) found differences in the strength of the alliance not only across treatment type but also across treatment settings (Mcleod et al., 2016). Specifically, observer-ratings of 
the alliance across time and adolescents' ratings at post-treatment were found to be higher in CBT in a research setting compared to both CBT and usual care in practice settings (McLeod et al., 2016). Based on these findings, there is some evidence that CBT may be associated with higher alliance with young patients than some other treatments. However, not all studies support this result, and no studies have investigated whether average levels of alliance in CBT differ from those in psychodynamic therapy for young people. Further, most studies do not differentiate between the specific alliance dimensions and investigating the trajectory of each alliance dimension separately might provide further insight into the relationship between alliance and treatment type.

There might not only be differences in the strength of the alliance and/or the way each treatment type emphasises different alliance dimension, but also in the way the alliance changes over time. Hatcher and Barends (2006) maintained that various therapy types place different emphasis on the alliance over the course of treatment. For instance, therapies that consider the alliance a prerequisite for the use of certain therapeutic techniques might focus more on fostering the alliance in the earlier phases of the therapy. In contrast, therapies that consider the alliance as a mechanism of change in itself might keep the same focus on the alliance throughout treatment. This might suggest that the trajectory of alliance over the course of treatment can vary as a function of therapeutic orientation. Yet, youth alliance research has focused predominantly on alliance measurement at discrete time points and rarely reports on comparisons of alliance strength across time in various types of treatments. Further, the small amount of research available on alliance trajectories in youth psychotherapy includes only one type of treatment (mostly CBT, non-specific treatments or aggregated treatments) and shows mixed findings. In particular, in CBT informed treatments, some found that the therapist- and adolescent- rated alliance initially increases and then levels off over time (Chu et al., 2014; Kendall et al., 2009). However, when using observer-rated measures, others found a slight 
decrease in the alliance over the course of CBT (Hudson et al., 2014; Liber et al., 2010; Mcleod et al., 2016). In psychodynamic therapy, only one study has investigated the trajectory of observer-rated mean alliance with children with internalising and externalising problems (Halfon et al., 2019). This study found a quadratic trend (high-low-high) in the alliance over the course of therapy. Based on existing evidence, it would be premature to draw firm conclusions about alliance trajectories over the course of treatment based on existing evidence. Further, these mixtures of results can be attributed to several methodological variations across studies including timing of alliance assessment, as well as different raters and setting.

It is plausible that various modalities of therapy might demand different kinds of goals and tasks, or a distinctive kind of bond, and that the alliance strength and trajectory might differ across therapy types. Yet there is a dearth of research on the topic and more research is needed to get further insight into the relationship between alliance and treatment.

\section{The current study}

This study attempts to clarify the relationship between alliance and treatment modalities in youth psychotherapy using data from the *anonymised* trial. The *anonymised* study was a randomised controlled trial (RCT) comparing the efficacy of $\mathrm{CBT}$ and short-term psychoanalytic psychotherapy (STPP) versus a brief psychosocial intervention (BPI) in the treatment of adolescent depression. These three treatments were found to be equally effective in maintaining a significant reduction in depressive symptoms one year after the end of treatment (*anonymised*, 2017).

The specific aims of this study were a) to assess whether the mean strength of adolescent- and therapist-rated alliance differed across three equally effective psychological treatments for adolescent depression; and b) to examine the mean trajectory of alliance change over time and explore whether there were differences in the alliance trajectories across treatment types; c) to investigate whether the findings from aim (a) and (b) regarding the overall 
alliance hold when looking at alliance components of Bond and Collaboration separately. Given the limited prior literature on the relationship between alliance and treatment type and competing theories about the impact of treatment type on alliance, we offer no a priori hypothesis about this relationship.

\section{Method}

The setting for this study is the *anonymised* trial, where 465 adolescents (aged between 11 and 17 years) with diagnosis of depression were randomised to receive either CBT, STPP or BPI (*anonymised*, 2017, 2011) at routine specialist child and adolescent mental health service (CAMHS) clinics in the UK. Full details of the method and procedure of the *anonymised* study are reported in *anonymised* and colleagues $(2011,2017)$. To maximize the number of participants, the present study included all IMPACT participants who received treatment and had at least one rating of the alliance completed by the adolescent or their therapist at any time in treatment.

\section{Ethical considerations}

The study protocol was approved by the *anonymised* ethics committee. Fully informed written consent was obtained from participants, or parents for those under the age of 16.

\section{Participants}

As participants' views of the alliance might differ, it is important not to combine alliance ratings from different raters. Therefore, participants in the study were divided into two samples based on whether the alliance was assessed from the adolescents' (Sample 1) or the therapists' (Sample 2). Samples descriptive and comparisons across treatment groups are displayed in Table 1 and described below.

Sample 1 (adolescents' alliance ratings). This sample consisted of 338 adolescents, $72.7 \%$ of the overall *anonymised* sample, and included all participants who completed one 
or more self-ratings of the alliance over time. Adolescents in this sample were treated by 157 therapists (BPI: $\mathrm{N}=69$; CBT: $N=49$; STPP: $N=39$ ).

Sample 2 (therapists' alliance ratings). This sample consisted of 159 adolescents, $34.2 \%$ of the overall *anonymised* sample, and included participants with at least one rating of the alliance completed by 72 therapists (BPI: $N=25$; CBT: $N=23$; STPP: $N=24)$.

As showed in Table 1 and similar to the overall *anonymised* sample (see Goodyer et al., 2017), across treatment arms there were no statistically significant differences in baseline age, sex and depression severity as well as in outcomes and mean duration of treatment. Therefore, excluding participants without alliance ratings did not seem to introduce bias with respect to these characteristics into the between-arm comparison, in line with the overall *anonymised* trial.

Therapists. No demographic information was collected for the therapists, so cannot be reported. Therapists were drawn from routine CAMHS clinics: CBT therapists were either clinical psychologists or had a qualification in CBT; STPP therapists were trained in child and adolescent psychoanalytical psychotherapy; and the majority of BPI therapists were child psychiatrists. All therapists received supervision. The median and mode of number of patients treated by each therapist was 1 , with only a few therapists treating more than one patient. Nesting withing therapist was nonetheless controlled for in the analyses, as described below. [Table 1 near here]

\section{Data Collection and Measures}

Demographics. Age, sex, and ethnicity were assessed with a demographic questionnaire at baseline.

Alliance measure. The Working Alliance Inventory short form (WAI-S) (Horvath \& Greenberg, 1989; Tracey \& Kokotovic, 1989) aims to measure Bordin's (1979) conceptualization of the working alliance. Accordingly, it includes three (4-item) subscales 
assessing: (a) agreement on Goals, (b) agreement on Tasks and (c) the emotional Bond between patient and therapist. Items are rated on a 7-point response scale (from 1=Never to 7=Always). The scale yields different ratings for each subscale as well as an aggregate overall rating ranging from 12 to 84 , with higher ratings reflecting a stronger alliance. Since in a previous study we found a lack of empirical evidence for the differentiation between Task and Goal (see *anonymised* et al. 2020), we used the overall alliance score and two subscales of Bond (score ranging from 4 to 28) and Collaboration (Task and Goal items combined, score ranging from 12 to 56 ). The WAI-S has demonstrated good validity and reliability (Horvath \& Greenberg, 1989; Tracey \& Kokotovic, 1989), as well as good internal consistency within youth samples (Capaldi et al., 2016; Dennis et al., 2002; Hawley \& Garland, 2008; Tetzlaff et al., 2005).

Both therapist (WAI-S-T) and patient (WAI-S) versions of the scale were collected at 6 weeks post-randomisation (within the first 4 sessions of treatment), 12 weeks (midtreatment), and 36 weeks (completed treatment for $69.9 \%$ of adolescents, late stage of treatment for $30.1 \%$ ) after randomization. These time points were pre-scheduled after randomization and do not necessarily correspond to the same number of sessions for all participants. However, information about how many sessions had been attended at each assessment point was not available.

Depression severity. The Mood and Feelings Questionnaire (MFQ) (Angold et al., 1987 ) is a 33-item self-report measure of depressive symptoms over the past two-week period. Total ratings range from 0 to 66 , with higher ratings reflecting higher depression severity. The clinical cut-off for the presence of a major depressive episode is 27 (Wood et al., 1995). In this study we report about depression severity at baseline and 1-year follow-up (86 weeks after randomization) to assess whether the adolescents included in this study had similar depression characteristics across treatment arms, like in the overall *anonymised* trial. 


\section{Treatment Arms}

Cognitive-Behavioural Therapy (CBT) focused on identifying the behaviours and the cognitive biases that maintain the depressive symptoms and aimed to amend these biases, through a process of collaborative empiricism between the therapist and patient. Sessions focused on working on explicit, tangible and shared goals (*anonymised*, 2010).

Short-Term Psychoanalytic Psychotherapy (STPP) aimed to promote better selfunderstanding of feelings and difficulties and to address the underlying dynamics of the symptoms, not only the symptoms per se (*anonymised*, 2016). STPP therapists focused on a close observation of the therapeutic relationship and used supportive and expressive strategies to address difficulties in the context of the developmental tasks of adolescence.

Brief Psychosocial Intervention (BPI) is a generic, goal-oriented treatment focused on psychoeducation about depression, problem solving, and encouraging increasing positive activities (*anonymised*, 2010). BPI was not designed to focus on self-understanding nor on changes in cognitions per se. This psychiatry-led approach emerged from the treatment as usual in the Adolescent Depression Antidepressants and Psychotherapy Trial (Wilkinson et al., 2011).

All treatments were manualised and demonstrated to be delivered with fidelity to their respective treatment approach (*anonymised*, 2017; *anonymised*, 2018). Some patients in all three groups also received antidepressant medication. Methods of prescribing did not differ between groups over the course of the study. In line with the overall *anonymised* sample, the length of treatment was equivalent across treatment arms (see Table 1).

\section{Data analysis}

The analyses of the effect of treatment condition on the mean alliance trajectory were conducted using multilevel modelling (MLM) with the "nlme" package (Pinheiro et al., 2019) of the R software (R Core Team, 2018). 
All models had a three-level structure with repeated alliance ratings (i.e. within-patient differences) at level 1, nested within participants (i.e. between-patient differences) at level 2, which in turn were nested within therapists (i.e. between-therapist differences) at level 3 . We allowed the intercept and the slope of time to vary randomly between both adolescents and therapists and used an unstructured covariance matrix for correlations between random effects. Group differences in mean alliance and change over time were modelled via the level 3-fixed effect predictor treatment arm, and the level 2 fixed effects predictor time, respectively. Since there were no differences in either demographic characteristics or depression severity at baseline across treatment arms, these variables were not controlled for in the analyses to keep the models as parsimonious as possible.

Treatment arm was treated as a categorical variable with STPP as reference group in the main model. Moreover, to allow for all pairwise comparisons across treatment groups, the same model was run again entering treatment arm with BPI as the reference group.

For the time predictor, firstly we identified the best way of modelling the time variable; then we assessed the interaction between the alliance trajectory and treatment type. To assess the shape of the mean alliance change over time we tested two models: 1) a discrete-time model of early, midpoint, and end of treatment assessment, where time was used as a categorical variable (6-, 12- and 36-weeks); and 2) a linear change model with time measured in weeks and centred at 6 weeks. Once optimal fit was determined for the time variable (discrete vs linear), we proceeded to examine whether there was an interaction between type of treatment and alliance change over time. Therefore, a two-way interaction term between time and arm (Model 2) was added to the best fitting model to assess whether the mean alliance change over time was dependent on treatment type. This model was then compared with the equivalent model without the interaction (Model 1) to assess whether adding the interaction improved model fit. 
For model comparisons we used the likelihood ratio (LR) tests, and the Akaike's Information Criterion (AIC) and Bayesian Information Criterion (BIC). Information criteria are indicators of model quality that take account of both model fit and model complexity, by penalising larger models. Smaller AIC and BIC indicate a better model.

All models were estimated separately for a) adolescent and therapist ratings, and b) the three different alliance (sub-)scales: (i) the WAI-S total scale, (ii) the Bond subscale, and (iii) the Collaboration subscale.

Missing data. Missing data at the WAI-S item level was handled using person-mean substitution when at least 9 out of 12 items of the scale were completed. If more than 3 items of the measure were missing, the data point (entire scale) was considered missing.

With regards to missing data at each time-point, within sample $1(\mathrm{n}=338), 78.5 \%$ of the adolescents rated the alliance at 2 or all 3 time points. The rate of missing WAI-S was $34.0 \%$ at 6 weeks, $26.9 \%$ at 12 weeks and $34.3 \%$ at 36 weeks. Similar to other self-report data collected as part of the *anonymised* trial, the cause of missing WAI-S was likely to be due to research attrition, time constraints in the assessments and questionnaire fatigue (*anonymised*, 2017). Therefore, missing data were assumed to be missing at random (Rubin, 1987) and handled using maximum likelihood (ML) estimation within the multilevel models (Gallop \& Tasca, 2009). This procedure takes into account information from all individuals in the sample when calculating parameter estimates, retaining patients in the longitudinal analysis who had at least one WAI-S score.

Within sample $2(n=159), 75.5 \%$ of the adolescents had rated the alliance at 2 or all 3 time points. The rate of missing therapist ratings (WAI-S-T) was $12.6 \%$ at 6 weeks, $25 \%$ at 12 weeks, and $60 \%$ at 36 weeks. Similar to sample 1, the amount of missing data in sample 2 was due to research attrition, but also treatment termination. While adolescents continued to be involved in the *anonymised* research up until 86 weeks post randomization, 36 weeks was 
the last research assessment for all therapist-rated measures. As such, in cases where therapy had ended before the 36-week mark, therapists were more difficult to contact as they were no longer involved in the trial. This might explain the higher percentage of missing at 36 weeks in sample 2 compared to sample 1. Although an advantage of the multilevel analyses lies in the flexibility in handling missing data (Gallop \& Tasca, 2009), the high amount of missing data at 36 weeks makes the estimates of the tail end of trajectories in this sample less reliable. Therefore, to assess whether missing data affected the results, a sensitivity analysis was conducted by undertaking the same analyses but including only complete cases (i.e., adolescents with therapists' ratings of the alliance at all three time-points).

\section{Results}

The observed mean alliance ratings for each treatment arm across time are presented in Table 2 and plotted in the Supplemental Figure 1 for both samples separately.

[Table 2 near here]

\section{Mean Alliance Trajectory}

Table S1 in the Supplemental material presents the comparison of the discrete-time model and the linear change model in both samples for all three WAI-S scales, separately. According to the fit indices and the Likelihood Ratio (LR) test, the linear model should be preferred to the discrete-time model in both samples for all alliance scales. This suggests that the overall alliance, as well the Bond and Collaboration subscales, showed a slight linear change over time, if it changed at all, from both the adolescent and therapist perspectives. Therefore, time was treated as linear in the subsequent analyses. The observed trajectories of youth- and therapist- rated alliance are displayed in the Supplemental Figures S2-S4.

\section{Effect of Treatment Condition on Alliance Mean Trajectory}

Table S2 in the Supplemental material shows the results of the comparison between the model including the interaction term between alliance and time (Model 2) and the simpler 
model that included time and arm as independent predictors of the alliance ratings (Model 1). For the adolescent ratings of the alliance (sample 1), according to likelihood ratio (LR) tests $(\mathrm{p}=0.030)$, as well as the AIC and BIC indices, the models including the interaction showed a better fit to the data compared to the competing model. This was true for all alliance ratings but was less marked for the Bond subscale. In contrast, for the therapists' ratings of the WAIS-T (sample 2) adding the interaction term did not improve model fit according to the LR tests $(p=0.966)$ and all fit indices for all alliance ratings.

As there was some evidence for the existence of different alliance trajectories across treatment arms from the adolescents', but not the therapists', alliance ratings, the below results present the model including the interaction term for sample 1, and the simple linear model for sample 2.

Adolescent ratings. As displayed in Table 3, there were significant differences in the overall alliance ratings across therapy groups. The mean alliance in the STPP arm at 6 weeks was lower than in both BPI and CBT. CBT showed the highest mean alliance ratings. On average the alliance showed to be relatively stable over time in both BPI and CBT. In contrast, in STPP there was statistical evidence of a slight $(b=0.15$ per week $)$ increase in the mean alliance from week 6 to week 36 . The left-hand side of Figure 1 shows the observed means and model predictions for the WAI-S overall alliance.

[Figure 1 near here]

Similar results were found for the Collaboration and the Bond subscales, with both alliance dimensions resulting significantly higher in CBT and BPI compared to STPP. There also was a greater increase in the mean alliance over time in STPP compared to the other treatment groups, although this was less marked for the Bond subscale.

[Table 3 near here] 
Therapist ratings. As displayed in Table 4, on average the overall alliance showed a linear increase over time. Mean alliance ratings differed between treatment types, being highest in the CBT arm and lowest in the STPP arm. The difference between BPI and CBT at 6 weeks was small $(b=1.14)$ and not statistically significant. The right-hand side of Figure 1 shows the observed means and model predictions for the WAI-S-T overall alliance. The observed ratings display a similar 'fanning in' effect to that observed in the adolescent ratings, whereby the difference between STPP and the other treatments is much smaller at 36 weeks than at 6 weeks. However, in the therapist ratings little statistical evidence for a difference in mean alliance change was found, as reported above (e.g. little evidence for an interaction between time and treatment type). The differences between the observed means and the predicted trajectories might be due to the large variability around the observed means and the larger proportion of missing values at 36 weeks compared to earlier time points (see Table 2).

[Table 4 near here]

Similar results were found for both the Bond and Collaboration subscales. Mean alliance ratings in both subscales were significantly lower in STPP compared to both BPI and CBT and showed a slight but significant increase over time across all therapies. The difference between the mean WAI-S-T Collaboration and Bond in BPI and CBT was small and not statistically significant ( $b=0.21$ for Collaboration and $b=1.11$ for the Bond subscale).

Sensitivity Analyses. Given the amount of missing data at 36 weeks in sample 2, a sensitivity analysis was conducted, undertaking the same analyses but using all cases that included the WAI-S-T at all three time points e.g., excluding cases with missing data. $(\mathrm{N}=51)$. The sensitivity analysis confirmed the findings on the overall sample 2. Details of the results of the sensitivity analyses are showed in table S3-S5 in the Supplemental material.

\section{Discussion}


This study examined this relationship in youth psychotherapy by investigating whether the mean alliance strength and its trajectory over the course of treatment differed between three therapeutic approaches for adolescent depression. While being equally effective (*anonymised*, 2017), the three treatments were associated with different mean alliance levels across time. Both adolescents' and therapists' ratings of the alliance were significantly lower in STPP when compared to BPI and especially CBT; which showed the highest alliance ratings. Although the average alliance was relatively stable over time for all treatment, we found some evidence that the adolescent-rated alliance trajectory might change differently over time depending on the treatment delivered. Despite starting from lower baseline alliance mean scores, the STPP group showed a greater increase in the alliance over time compared to the other two treatment groups. BPI and CBT, instead, both showed an approximately stable alliance pattern over time. Hence, the differences of the alliance ratings across treatments were more evident in earlier phases of treatment and decreased slightly at the end of treatment. From the therapist perspective, although the observed increase in the mean alliance over time was more pronounced in STPP than in CBT and BPI, there was no strong evidence of the existence of differences in the alliance trajectory across treatment types. This result might be due to the lack of statistical power in this sample, given its small sample size and the higher proportion of missing data compared with the sample including adolescent ratings of the alliance.

Importantly, in our study there were no clear differences in the strength and patterns of the overall alliance ratings compared to the ones of the two subscales of Bond and Collaboration across time, treatment arm and rater perspective. One explanation for this could be that the specific alliance components follow a similar pattern across time and treatment type and therefore cannot be disentangled. This might be because it is difficult to establish a good collaboration without a strong bond and, vice-versa, a positive emotional bonding is essential for the patient's motivation and involvement in therapy. Alternatively, this result could be due 
to methodological issues related to the alliance measure used in this study. It could be argued that the WAI is not able to meaningfully differentiate between these two alliance components due to the high intercorrelation between the WAI subscales ("anonymised"; Falkenström et al., 2015). This result would support the findings of previous factor analytic studies on the WAI, which suggest the use of the overall alliance score only ("anonymised"; Falkenström et al., 2015).

Overall, these findings could suggest that even if the alliance is a common factor, its mean strength and trajectory over time may vary across therapy types for depressed adolescents. However, more research is needed to confirm our findings and test whether various types of treatments, when offered to comparable patient groups, have similar or different alliance ratings over time. To date, the available literature on the topic is limited, presents mixed findings and it is mainly based on comparison between studies using various types of therapy, diverse samples and different alliance raters (Chu et al., 2014; Halfon et al., 2019; Kendall et al., 2009). Since this is the first study to compare the alliance in manualised psychodynamic therapies and CBT for young people, we cannot compare our results with previous research. Instead, we can make sense of our findings using theoretical and methodological considerations.

On a theoretical level, the overall differences in the strength of the alliance across therapies found in this study might support the idea that the quality of the alliance differs across therapeutic approaches (Bordin, 1979; Hatcher \& Barends, 2006; Wampold \& Budge, 2012). Specifically, we found that CBT was associated with adolescent's and therapists' higher mean alliance ratings (overall, as well as Bond and Collaboration) compared to STPP. This result might be due to the emphasis that CBT places on the idea of having explicit discussions about therapy goals and tasks throughout therapy to reach a 'collaborative empiricism', which might help to foster a strong alliance from the onset of therapy (Tee \& Kazantzis, 2011). In contrast, 
in the STPP manual (*anonymised*, 2016), there is no specific suggestion that tasks and goals should be explicitly discussed during sessions. This might restrict the development of a strong collaboration around tasks and goals in this therapy group and might be responsible for the lower alliance ratings reported by both adolescent and therapists. Furthermore, the STPP manual focuses on the importance of establishing trust and a secure base, while also giving considerable attention to allowing negative feelings to enter the relationship, via the negative transference. Working with the negative transference refers to the therapist's capacity to acknowledge and facilitate the emotional expression of the young person's negative feelings in relation to the therapist, and to be able to tolerate them, rather than avoid or hide them. Working through painful and hostile feelings could be difficult and frustrating for the young person and "may appear to indicate a breakdown in the therapeutic alliance" (Cregeen et al, 2016; $p$ 53) and therefore be responsible for the lower alliance ratings found in this treatment arm. The alliance strength in BPI was slightly lower than CBT but still higher than STPP. While the activities and goals of BPI were different from those of CBT, tasks and goals were supposed to be discussed in this treatment condition too. The BPI manual emphasised the importance of psychoeducation about depression and the development of jointly agreed management plan and goals action-orientated activities (Kelvin et al., 2010). This aspect of BPI might, therefore, also have facilitated the development of an alliance between patient and therapist in this treatment group.

The results of this study could also be in part related to the conceptualization and measurement of the alliance used in this study. It could be argued that Bordin's definition of the alliance might be more easily applied to CBT and to therapies like BPI, where encouraging explicit collaboration on tasks and setting common goals are essential components and a regular focus of therapy sessions (*anonymised*; Kelvin et al., 2010), than to psychodynamic therapies. Psychodynamic therapies do not aim to set 'tasks' and 'goals', and theoretical 
accounts of the alliance in these therapies don't always refer to Bordin's concept. For instance, the STPP Manual refers to Luborsky's (1976) ideas about two types of alliance: one "based on the patient's experiencing the therapist as supportive and helpful" and one "based on a sense of working together in a joint struggle" (p. 94). Based on this rationale, the language used in the WAI-S items might better capture the way the alliance is described and used in CBT, and perhaps BPI, compared to STPP. Not surprisingly, it has been argued that Bordin's (1979) alliance concept, which was the conceptual basis for the WAI, does not fully address the complexities of the emotional relationship between patient and therapist in psychodynamic treatments (Castonguay et al., 2006).

Beyond demonstrating that treatment type might impact on the alliance strength and pattern, our findings contribute to a growing body of research suggesting that in youth psychotherapy the mean alliance strength remains more or less stable over time in CBT and similar treatments, especially when measured by young people. The effect of time was stronger from the therapist than the adolescent perspective, with therapist-rated alliance showing a slight increase over time. Previous research also found more variability in the way therapists rate the alliance compared to adolescents (Bickman et al., 2012; Chu et al., 2014). These results are also in line with other research showing that mean youth ratings of the alliance tend to be stable (Accurso \& Garland, 2015; Chu et al., 2014), or increase slightly over time (Bickman et al., 2012; Kendall et al., 2009). However, the overall stability of the alliance over time might also be related to methodological issues related to the use of self-report measures of the alliance, especially with youths and the limited number of alliance assessments over time. Self-report measures are commonly prone to response distortions, including those of acquiescence, social desirability and extreme response sets (DeVellis, 2016).

Even if the aim of this study was not to link the alliance to outcome, it is important to notice that despite the differences in the alliance levels across treatment types, the three 
treatments were found to be equally effective. This is in line with research suggesting that lower alliance ratings in some stages of therapy are not necessarily an indication of unsuccessful therapy (Eubanks et al., 2018). If alliance strains are addressed and resolved in therapy, they might lead to improved alliance and good outcomes (Muran \& Safran, 2016). As such, the increase of the alliance over time in the STPP arm found in this study might indicate the resolution of alliance ruptures which in turn lead to better alliance over time. Lower global alliance ratings might be a necessary element of some treatments or some stages of treatments and may or may not impact on treatment outcome. Of note, these are just speculations and further research is needed to understand the nature and role of these differences and their links to outcomes.

\section{Strengths and Limitations}

This study has several strengths including being the first study to evaluate whether on average the alliance strength differs across three different treatments for adolescent depression, and to examine whether alliance trajectory changed differently over time depending on what treatment was delivered. Furthermore, data were derived from a randomized controlled trial in which the adherence to treatments was closely monitored for integrity. Randomization means that treatment selection bias and other confounding variables can be ruled out as explanations for our findings. Other strengths of this study were the inclusion both adolescents' and therapists' ratings and the differentiation between the specific alliance dimensions of bond and collaboration.

An important limitation of this study was that the alliance assessment was restricted to three time points rather than after each therapy session. This limited our ability to assess more complex alliance trajectories/pattern and therefore the possibility to assess sophisticated dynamics in alliance trajectories over time (Chu et al., 2014; Kivlighan \& Shaughnessy, 1995). Additionally, alliance ratings were not completed after a specific treatment session, but at 
scheduled time-points post-randomization, as part of the overall *anonymised* trial. These time points do not correspond precisely to the same number of sessions for all participants, and at the last assessment most participants had completed therapy. Furthermore, the number of therapists' ratings of the alliance was small and there was a greater proportion of missing data toward the end of therapy, which makes estimates of the tail end of trajectories less reliable and precise. Although the sensitivity analysis confirmed the result on the overall sample, a larger sample size may have given greater power to detect alliance trajectories or interaction effect for the therapists' ratings.

\section{Conclusion}

The results of the present study provide evidence for the existence of differences in the mean alliance strength across three therapy types, which were shown to be equally effective for treating adolescent depression in the clinical trial from which this data was taken. This might support the assumption that the techniques used by different therapeutic approaches are "intrinsically bound to the relationship context in which they are applied" (Norcross \& Lambert, 2014). Therefore, reference to the alliance as a common factor may be misleading in the sense that the alliance strength and the manner in which it interacts with the specific treatment type to achieve benefits may not be the same across therapies (Hoffart et al., 2012). It is also possible that widely used measures of the therapeutic alliance, such as the WAI, have a better conceptual fit with some types of therapy than others. Future research should seek to further understand the relationship between alliance and treatment type and what factors contribute to certain treatments achieving better alliance ratings than others. Furthermore, it will be important to examine whether any differences in mean alliance ratings or trajectories of alliance over time are associated with differences in outcomes.

\section{References}

Accurso, E. C., \& Garland, A. F. (2015). Child, caregiver, and therapist perspectives on 
therapeutic alliance in usual care child psychotherapy. Psychol Assess, 27(1), 347-352. https://doi.org/10.1037/pas0000031

Anderson, R. E. E., Spence, S. H., Donovan, C. L., March, S., Prosser, S., \& Kenardy, J. (2012). Working alliance in online cognitive behavior therapy for anxiety disorders in youth: Comparison with clinic delivery and its role in predicting outcome. Journal of Medical Internet Research, 14(3), e88. https://doi.org/10.2196/jmir.1848

Angold, A., Costello, E. J., Pickles, A., Winder, F., \& Silver, D. (1987). The development of a questionnaire for use in epidemiological studies of depression in children and adolescents. London: Medical Research Council Child Psychiatry Unit.

Bickman, L., Regina, A., Andrade, V. De, Athay, M. M., Chen, J. I., \& Karver, M. S. (2012). The relationship between change in therapeutic alliance ratings and improvement in youth symptom severity: Whose ratings matter the most? Administration and Policy in Mental Health and Mental Health Services Research, 39(1-2), 78-89. https://doi.org/10.1007/s10488-011-0398-0.

Bordin, E. S. (1979). The generalizability of the psychoanalytic concept of the working alliance. Psychotherapy: Theory, Research \& Practice, 16(3), 252-260.

Capaldi, S., Asnaani, A., Zandberg, L. J., Carpenter, J. K., \& Foa, E. B. (2016). Therapeutic Alliance during Prolonged Exposure Versus Client-Centered Therapy for Adolescent Posttraumatic Stress Disorder. Journal of Clinical Psychology, 72(10), 1026-1036.

Castonguay, L. G., Constantino, M. J., \& Holtforth, M. G. (2006). The working alliance: Where are we and where should we go? Psychotherapy, 43(3), 271-279. https://doi.org/10.1037/0033-3204.43.3.271

Chu, B. C., Skriner, L. C., \& Zandberg, L. J. (2014). Trajectory and Predictors of Alliance in Cognitive Behavioral Therapy for Youth Anxiety. Journal of Clinical Child \& Adolescent Psychology, 43(5), 721-734. https://doi.org/10.1080/15374416.2013.785358

Cirasola, A., Midgley, N., Fonagy, P., Consortium, I., \& Martin, P. (2020). The factor structure of the Working Alliance Inventory short-form in youth psychotherapy : an empirical investigation youth psychotherapy : an empirical investigation. Psychotherapy Research, O(0), 1-13. https://doi.org/10.1080/10503307.2020.1765041 
Dennis, M. L., Titus, J. C., Diamond, G. S., Donaldson, J., Godley, S. H., Tims, F. M., Webb, C., Kaminer, Y., Babor, T., Roebuck, M. C., Godley, M. D., Hamilton, N., Liddle, H., \& Scott, C. K. (2002). The Cannabis Youth Treatment (CYT) experiment: Rationale, study design and analysis plans. In Addiction (Vol. 97, Issue SUPPL. 1). UCHC. https://doi.org/10.1046/j.1360-0443.97.s01.2.x

DeRubeis, R. J., Brotman, M. A., \& Gibbons, C. J. (2005). A conceptual and methodological analysis of the nonspecifics argument. Clinical Psychology: Science and Practice, 12(2), $174-183$.

DeVellis, R. F. (2016). Scale development: Theory and applications (Vol. 26). Sage publications.

Diamond, G. M., Hogue, A., Liddle, H. A., \& Dakof, G. A. (1999). Alliance-building interventions with adolescents in family therapy: A process study. Psychotherapy, 36(4), $355-368$.

Eubanks, C. F., Muran, C. J., \& Safran, J. D. (2018). Alliance Rupture Repair: A MetaAnalysis. Psychotherapy, 55(4), 508-519. https://doi.org/10.1037/pst0000185.supp

Falkenström, F., Hatcher, R. L., \& Holmqvist, R. (2015). Confirmatory Factor Analysis of the Patient Version of the Working Alliance Inventory-Short Form Revised. Assessment, 22(5), 581-593. https://doi.org/10.1177/1073191114552472

Faw, L., Hogue, A., Johnson, S., Diamond, G. M., \& Liddle, H. A. (2005). The Adolescent Therapeutic Alliance Scale (ATAS): Initial psychometrics and prediction of outcome in family-based substance abuse prevention counseling. Psychotherapy Research, 15(1-2), 141-154. https://doi.org/10.1080/10503300512331326994

Fjermestad, K. W., McLeod, B. D., Heiervang, E. R., Havik, O. E., Öst, L. G., \& Haugland, B. S. M. (2012). Factor Structure and Validity of the Therapy Process Observational Coding System for Child Psychotherapy-Alliance Scale. Journal of Clinical Child and Adolescent Psychology, 41(2), 246-254. https://doi.org/10.1080/15374416.2012.651999

Freud, A. (1946). The psycho-analytical treatment of children. Imago Publishing Co. http://psycnet.apa.org/record/1947-01846-000 
Gallop, R., \& Tasca, G. A. (2009). Multilevel modeling of longitudinal data for psychotherapy researchers: II. The complexities. Psychotherapy Research, 19(4-5), $438-452$.

Halfon, S., Ozsoy, D., \& Cavdar, A. (2019). Therapeutic Alliance Trajectories and Associations with Outcome in Psychodynamic Child Psychotherapy. Journal of Consulting and Clinical Psychology, 87(7), 603-616. https://doi.org/10.1037/ccp0000415

Hartmann, A., Joos, A., Orlinsky, D. E., Zeeck, A., Hartmann, A., Joos, A., Orlinsky, D. E., \& Zeeck, A. (2015). Accuracy of therapist perceptions of patients' alliance : Exploring the divergence the divergence. Psychotherapy Research, 25(4), 408-419. https://doi.org/10.1080/10503307.2014.927601

Hatcher, R. L. (2010). Alliance Theory and Measurment. In C. J. Muran \& P. Barber, Jacques (Eds.), The Therapeutic Alliance: An Evidence-Based Guide to Practice (pp. 7-28). The Guilford Press. https://www.guilford.com/excerpts/muran.pdf?t

Hatcher, R. L., \& Barends, A. W. (2006). How a return to theory could help alliance research. Psychotherapy, 43(3), 292-299. https://doi.org/10.1037/0033-3204.43.3.292

Hawley, K. M., \& Garland, A. F. (2008). Working alliance in adolescent outpatient therapy: Youth, parent and therapist reports and associations with therapy outcomes. Child and Youth Care Forum, 37(2), 59-74. https://doi.org/10.1007/s10566-008-9050-x

Hoffart, A., Borge, F.-M., Sexton, H., Clark, D. M., \& Wampold, B. E. (2012). Psychotherapy for social phobia: How do alliance and cognitive process interact to produce outcome? Psychotherapy Research, 22(1), 82-94.

Hogue, A., Dauber, S., Stambaugh, L. F., Cecero, J. J., Howard, L. A., \& Liddle, H. A. (2006). Early Therapeutic Alliance and Treatment Outcome in Individual and Family Therapy for Adolescent Behavior Problems Aaron. J Consult Clin Psychol, 74(1), 121129. https://doi.org/10.1037/0022-006X.74.1.121.

Horvath, A. O. (2018). Research on the alliance: Knowledge in search of a theory. Psychotherapy Research, 28(4), 499-516. 
Horvath, A. O., \& Greenberg, L. S. (1989). Development and validation of the Working Alliance Inventory. Journal of Counseling Psychology, 36(2), 233.

Hougaard, E. (1994). The therapeutic alliance-A conceptual analysis. Scandinavian Journal of Psychology, 35(1), 67-85.

Hudson, J. L., Kendall, P. C., Chu, B. C., Gosch, E., Martin, E., Taylor, A., \& Knight, A. (2014). Child involvement, alliance, and therapist flexibility: Process variables in cognitive-behavioural therapy for anxiety disorders in childhood. Behaviour Research and Therapy, 52(1). https://doi.org/10.1016/j.brat.2013.09.011

IMPACT Study CBT Sub-Group. (2010). Cognitive behaviour therapy for depression in young people: Manual for therapists. http://dev.psychiatry.cam.ac.uk/projects/

Karver, M. S., De Nadai, A. S., Monahan, M., \& Shirk, S. R. (2018). Meta-analysis of the prospective relation between alliance and outcome in child and adolescent psychotherapy. Psychotherapy, 55(4), 341-355. https://doi.org/10.1037/pst0000176

Kelvin, R. G., Dubicka, B., Wilkinson, P. O., \& Goodyer, I. M. (2010). Brief Psychosocial Intervention (BPI): A specialist clinical care treatment manual for CAMHS use. In Cambridge: University of Cambridge. (Ed.), Cambridge: University of Cambridge (2010).

Kendall, P. C., Comer, J. S., Marker, C. D., Creed, T. A., Puliafico, A. C., Hughes, A. A., Martin, E. D., Suveg, C., \& Hudson, J. L. (2009). In-Session Exposure Tasks and Therapeutic Alliance Across the Treatment of Childhood Anxiety Disorders. Journal of Consulting and Clinical Psychology, 77(3), 517-525. https://doi.org/10.1037/a0013686

Kivlighan, D. M., \& Shaughnessy, P. (1995). Analysis of the Development of the Working Alliance Using Hierarchical Linear Modeling. Journal of Counseling Psychology, 42(3), 338-349. https://doi.org/10.1037/0022-0167.42.3.338

Langer, D. A., McLeod, B. D., \& Weisz, J. R. (2011). Do treatment manuals undermine youth-therapist alliance in community clinical practice? Journal of Consulting and Clinical Psychology, 79(4), 427-432. https://doi.org/10.1037/a0023821

Liber, J. M., McLeod, B. D., Van Widenfelt, B. M., Goedhart, A. W., van der Leeden, A. J. 
M., Utens, E. M. W. J., \& Treffers, P. D. A. (2010). Examining the Relation Between the Therapeutic Alliance, Treatment Adherence, and Outcome of Cognitive Behavioral Therapy for Children With Anxiety Disorders. Behavior Therapy, 41(2), 172-186. https://doi.org/10.1016/j.beth.2009.02.003

Luborsky, L. (1976). Helping alliances in psychotherapy: The groundwork for a study of their relationship to its outcome. Successful Psychotherapy, 92-116.

Marmar, C. R., Gaston, L., Gallagher, D., \& Thompson, L. W. (1989). Alliance and outcome in late-life depression. Journal of Nervous and Mental Disease.

McLeod, B. D. (2011). Relation of the alliance with outcomes in youth psychotherapy: A meta-analysis. Clinical Psychology Review, 31(4), 603-616. https://doi.org/10.1016/j.cpr.2011.02.001

Mcleod, B. D., Jensen-Doss, A., Tully, C. B., Southam-Gerow, M. A., Weisz, J. R., \& Kendall, P. C. (2016). The Role of Setting Versus Treatment Type in Alliance within Youth Therapy. J Consult Clin Psychol, 84(5), 453-464. https://doi.org/10.1037/ccp0000081

Meyer, B., Pilkonis, P. A., Krupnick, J. L., Egan, M. K., Simmens, S. J., \& Sotsky, S. M. (2002). Treatment Expectancies, Patient Alliance, and Outcome: Further Analyses From the National Institute of Mental Health Treatment of Depression Collaborative Research Program. https://doi.org/10.1037/0022-006X.70.4.1051

Muran, C. J., \& Barber, J. P. (2010). The therapeutic alliance: An evidence-based approach to practice and training. New York: Guilford.

Muran, C. J., \& Safran, J. (2016). Therapeutic alliance ruptures. Sage Encyclopedia of Abnormal \& Clinical Psychology, JANUARY.

Norcross, J. C., \& Lambert, M. J. (2014). Relationship science and practice in psychotherapy: Closing commentary. Psychotherapy, 51(3), 398-403. https://doi.org/10.1037/a0037418

Ormhaug, S. M., Jensen, T. K., Wentzel-Larsen, T., \& Shirk, S. R. (2014). The therapeutic alliance in treatment of traumatized youths: Relation to outcome in a randomized clinical trial. Journal of Consulting and Clinical Psychology, 82(1), 52-64. 
https://doi.org/10.1037/a0033884

Pinheiro, J., Bades, D., DebRoy, S., Sarkar, D., \& R Core Team. (2019)._nlme: Linear and Nonlinear Mixed Effects Models_. R package version 3.1-140.

R Core Team. (2018). R: A language and environment for statistical computing. $R$ Foundation for Statistical Computing, Vienna, Austria. https://www.r-project.org/

Raue, P. J., \& Goldfried, M. R. (1994). The therapeutic alliance in cognitive-behavior therapy. The Working Alliance: Theory, Research, and Practice, 173, 131-152.

Raue, P. J., Goldfried, M. R., \& Barkham, M. (1997). The therapeutic alliance in psychodynamic-interpersonal and cognitive-behavioral therapy. Journal of Consulting and Clinical Psychology, 65(4), 582.

Rubin, D. B. (1987). Multiple imputation for survey nonresponse. New York: Wiley.

Shelef, K., \& Diamond, G. M. (2008). Short form of the revised vanderbilt therapeutic alliance scale: Development, reliability, and validity. Psychotherapy Research, 18(4), 433-443. https://doi.org/10.1080/10503300701810801

Shelef, K., Diamond, G. M., Diamond, G. S., \& Liddle, H. A. (2005). Adolescent and Parent Alliance and Treatment Outcome in Multidimensional Family Therapy. Journal of Consulting and Clinical Psychology, 73(4). https://doi.org/10.1037/0022-006X.73.4.689

Shirk, S. R., Karver, M. S., \& Brown, R. (2011a). The Alliance in Child and Adolescent Psychotherapy. Psychotherapy, 48(1), 17-24. https://doi.org/10.1037/a0022181

Shirk, S. R., Karver, M. S., \& Brown, R. (2011b). The Alliance in Child and Adolescent Psychotherapy. Psychotherapy, 48(1), 17-24. https://doi.org/10.1037/a0022181

Shirk, S. R., \& Saiz, C. C. (1992). Clinical, empirical, and developmental perspectives on the therapeutic relationship in child psychotherapy. Development and Psychopathology, 4(4), 713-728. https://doi.org/10.1017/S0954579400004946

Tee, J., \& Kazantzis, N. (2011). Collaborative Empiricism in Cognitive Therapy: A Definition and Theory for the Relationship Construct. Clinical Psychology: Science and Practice, 18(1), 47-61. https://doi.org/10.1111/j.1468-2850.2010.01234.x 
Tetzlaff, B. T., Kahn, J. H., Godley, S. H., Godley, M. D., Diamond, G. S., \& Funk, R. R. (2005). Working alliance, treatment satisfaction, and patterns of posttreatment use among adolescent substance users. Psychology of Addictive Behaviors, 19(2), 199-207. https://doi.org/10.1037/0893-164X.19.2.199

Tracey, T. J., \& Kokotovic, A. M. (1989). Factor Structure of the Working Alliance Inventory. In Psychological Assessment: A Journal of Consulting and Clinical Psychology (Vol. 1, Issue 3).

Ulvenes, P. G., Berggraf, L., Hoffart, A., Stiles, T. C., Svartberg, M., McCullough, L., \& Wampold, B. E. (2012). Different processes for different therapies: Therapist actions, therapeutic bond, and outcome. Psychotherapy, 49(3), 291-302. https://doi.org/10.1037/a0027895

Wampold, B. E., \& Budge, S. L. (2012). The 2011 Leona Tyler Award Address: The relationship - And its relationship to the common and specific factors of psychotherapy. The Counseling Psychologist, 40(4), 601-623.

Webb, C. A., Derubeis, R. J., Amsterdam, J. D., Shelton, R. C., Hollon, S. D., \& Dimidjian, S. (2011). Two aspects of the therapeutic alliance: Differential relations with depressive symptom change. Journal of Consulting and Clinical Psychology, 79(3), 279-283. https://doi.org/10.1037/a0023252

Wilkinson, P., Kelvin, R., Roberts, C., Dubicka, B., \& Goodyer, I. (2011). Clinical and psychosocial predictors of suicide attempts and nonsuicidal self-injury in the Adolescent Depression Antidepressants and Psychotherapy Trial (ADAPT). American Journal of Psychiatry, 168(5), 495-501.

Wintersteen, M. B., Mensinger, J. L., \& Diamond, G. S. (2005). Do gender and racial differences between patient and therapist affect therapeutic alliance and treatment retention in adolescents? Professional Psychology: Research and Practice, 36(4), 400408. https://doi.org/10.1037/0735-7028.36.4.400

Wood, A., Kroll, L., Moore, A., \& Harrington, R. (1995). Properties of the mood and feelings questionnaire in adolescent psychiatric outpatients: a research note. Journal of Child Psychology and Psychiatry, 36(2), 327-334. 
Zandberg, L. J., Skriner, L. C., \& Chu, B. C. (2015). Client-therapist alliance discrepancies and outcome in cognitive-behavioral therapy for youth anxiety. Journal of Clinical Psychology, 71(4), 313-322. https://doi.org/10.1002/jclp.22167

Zilcha-Mano, S., Roose, S. P., Brown, P. J., \& Rutherford, B. R. (2019). Not just nonspecific factors: The roles of alliance and expectancy in treatment, and their neurobiological underpinnings. Frontiers in Behavioral Neuroscience, 12(January), 1-8. https://doi.org/10.3389/fnbeh.2018.00293 
Table 1. Sample descriptive and comparison across groups

\begin{tabular}{|c|c|c|c|c|c|c|c|c|}
\hline \multicolumn{9}{|c|}{ WAI-S sample $(\mathrm{N}=338)$} \\
\hline & \multicolumn{2}{|c|}{ BPI $(n=114)$} & \multicolumn{2}{|c|}{$\operatorname{CBT}(n=114)$} & \multicolumn{2}{|c|}{ STPP $(n=110)$} & \multirow[b]{2}{*}{ Chi2 } & \multirow[b]{2}{*}{$\mathbf{p}$} \\
\hline & $\mathbf{N}$ & $\%$ & $\mathbf{N}$ & $\%$ & $\mathbf{N}$ & $\%$ & & \\
\hline Gender & & & & & & & 0.59 & 0.744 \\
\hline Male & 31 & 27.2 & 28 & 24.6 & 32 & 29.1 & & \\
\hline Female & 83 & 72.8 & 86 & 75.4 & 78 & 70.1 & & \\
\hline Ethnicity & & & & & & & 0.35 & 0.841 \\
\hline White British & 85 & 74.6 & 90 & 78.9 & 86 & 78.2 & & \\
\hline \multirow[t]{2}{*}{ Other } & 25 & 21.9 & 23 & 20 & 21 & 19.1 & & \\
\hline & Mean & SD & Mean & SD & Mean & SD & $\mathbf{F}$ & $\mathbf{p}$ \\
\hline Age & 15.56 & 1.37 & 15.63 & 1.39 & 15.6 & 1.48 & 0.09 & 0.918 \\
\hline \multicolumn{9}{|l|}{ Depression Severity } \\
\hline MFQ at baseline & 47.19 & 9.96 & 46.16 & 10.3 & 44.3 & 10.7 & 2.19 & 0.916 \\
\hline MFQ at 86 weeks & 22.64 & 16.18 & 21.53 & 15.3 & 20.8 & 14.7 & 0.35 & 0.705 \\
\hline Weeks in treatment & 28.89 & 21.09 & 26.54 & 17.3 & 29.7 & 16.8 & 0.99 & 0.371 \\
\hline \multicolumn{9}{|c|}{ WAI-S-T sample $(\mathrm{N}=159)$} \\
\hline & \multicolumn{2}{|c|}{ BPI $(n=41)$} & \multicolumn{2}{|c|}{ CBT $(n=49)$} & \multicolumn{2}{|c|}{ STPP $(n=69)$} & \multirow[b]{2}{*}{ Chi2 } & \multirow[b]{2}{*}{$\mathbf{p}$} \\
\hline & $\mathbf{N}$ & $\%$ & $\mathbf{N}$ & $\%$ & $\mathbf{N}$ & $\%$ & & \\
\hline Gender & & & & & & & 1.82 & 0.401 \\
\hline Male & 16 & 39 & 13 & 26.5 & 20 & 29 & & \\
\hline Female & 25 & 61 & 36 & 73.5 & 49 & 71 & & \\
\hline Ethnicity & & & & & & & 2.16 & 0.339 \\
\hline White British & 30 & 73.2 & 32 & 65.3 & 52 & 74.5 & & \\
\hline \multirow[t]{2}{*}{ Other } & 11 & 26.8 & 17 & 34.7 & 15 & 21.7 & & \\
\hline & Mean & SD & Mean & SD & Mean & SD & $\mathbf{F}$ & $\mathbf{p}$ \\
\hline Age & 15.56 & 1.37 & 15.63 & 1.39 & 15.6 & 1.48 & 2.11 & 0.125 \\
\hline \multicolumn{9}{|l|}{ Depression Severity } \\
\hline MFQ at baseline & 46.34 & 11.54 & 46.85 & 11.3 & 45.8 & 10 & 0.13 & 0.878 \\
\hline MFQ at 86 weeks & 22.60 & 15.23 & 21.83 & 14.1 & 19.6 & 14.2 & 0.55 & 0.576 \\
\hline Weeks in treatment & 28.78 & 14.81 & 26.86 & 13.7 & 30.4 & 14.5 & 3.00 & 0.055 \\
\hline
\end{tabular}

Note: $\mathrm{MFQ}=$ The Mood and Feelings Questionnaire 
Table 2. Mean WAI-S scores at each time point, for both samples in each treatment arm

\begin{tabular}{ccccc|ccc|ccc}
\hline & Time & \multicolumn{3}{c}{ BPI $(\mathrm{n}=114)$} & \multicolumn{3}{c}{ CBT $(\mathrm{n}=114)$} & \multicolumn{3}{c}{ STPP $(\mathrm{n}=110)$} \\
\cline { 3 - 12 } WAI-S sample & week & $\mathbf{N}$ & Mean & SD & N & Mean & SD & N & Mean & SD \\
\hline Collaboration & 6 & 72 & 39.93 & 9.52 & 78 & 42.83 & 8.17 & 73 & 32.44 & 12.32 \\
& 12 & 84 & 37.88 & 8.98 & 82 & 42.71 & 8.95 & 81 & 32.07 & 12.48 \\
& 36 & 70 & 39.99 & 11.17 & 78 & 41.32 & 10.41 & 74 & 36.11 & 13.22 \\
\hline Bond & 6 & 72 & 19.60 & 5.25 & 78 & 20.47 & 4.89 & 73 & 15.90 & 7.19 \\
& 12 & 84 & 18.79 & 5.50 & 82 & 20.73 & 5.69 & 81 & 15.73 & 6.82 \\
& 36 & 70 & 19.28 & 6.64 & 78 & 20.29 & 6.14 & 74 & 17.78 & 6.75 \\
\hline Overall Alliance & 6 & 72 & 59.54 & 13.84 & 78 & 63.30 & 11.97 & 73 & 48.34 & 19.04 \\
& 12 & 84 & 56.67 & 13.72 & 82 & 63.44 & 14.06 & 81 & 47.80 & 18.71 \\
WAI-S-T sample & 36 & 70 & 59.27 & 17.25 & 78 & 61.61 & 16.07 & 74 & 53.89 & 19.49 \\
\hline Collaboration & 6 & 32 & 41.59 & 7.77 & 46 & 38.11 & 8.07 & 61 & 32.99 & 6.98 \\
& 12 & 32 & 38.34 & 9.25 & 34 & 41.56 & 8.41 & 53 & 34.04 & 8.25 \\
& 36 & 18 & 43.44 & 8.62 & 17 & 43.29 & 6.77 & 28 & 39.33 & 7.77 \\
\hline Bond & 6 & 32 & 21.69 & 3.61 & 46 & 20.80 & 3.30 & 61 & 18.21 & 3.23 \\
& 12 & 32 & 20.28 & 4.07 & 34 & 22.12 & 3.17 & 53 & 19.03 & 3.41 \\
& 36 & 18 & 21.94 & 3.02 & 17 & 23.92 & 1.56 & 28 & 21.13 & 3.41 \\
\hline Overall Alliance & 6 & 32 & 63.28 & 10.97 & 46 & 58.91 & 11.06 & 61 & 51.19 & 9.65 \\
& 12 & 32 & 58.63 & 13.11 & 34 & 63.68 & 11.22 & 53 & 53.07 & 11.20 \\
& 36 & 18 & 65.39 & 11.14 & 17 & 67.22 & 8.03 & 28 & 60.46 & 10.72 \\
\hline
\end{tabular}

Table 3. Estimates from multilevel models predicting adolescent ratings of the WAI-S: Total score, Collaboration Subscale, and Bond Subscale.

\begin{tabular}{|c|c|c|c|c|c|c|c|c|c|}
\hline \multicolumn{4}{|c|}{ WAI-S overall } & \multicolumn{3}{|c|}{ WAI-S Collaboration } & \multicolumn{3}{|c|}{ WAI-S Bond } \\
\hline Variables & Estimate & SE & $\mathbf{p}$ & Estimate & $\mathbf{S E}$ & $\mathbf{p}$ & Estimate & SE & $\mathbf{p}$ \\
\hline \multicolumn{10}{|l|}{ Fixed effect } \\
\hline Intercept & 48.44 & 1.54 & 0.000 & 32.31 & 0.99 & 0.000 & 16.08 & 0.60 & 0.000 \\
\hline Time & 0.15 & 0.06 & 0.017 & 0.11 & 0.04 & 0.010 & 0.04 & 0.02 & 0.069 \\
\hline \multicolumn{10}{|l|}{ Arm (ref: STPP) } \\
\hline Arm: BPI & 8.94 & 2.14 & 0.000 & 6.11 & 1.40 & 0.000 & 2.90 & 0.83 & 0.001 \\
\hline Arm: CBT & 14.72 & 2.13 & 0.000 & 10.31 & 1.39 & 0.000 & 4.45 & 0.83 & 0.000 \\
\hline Time*BPI & -0.14 & 0.09 & 0.104 & -0.10 & 0.06 & 0.860 & -0.04 & 0.03 & 0.205 \\
\hline Time*CBT & -0.23 & 0.08 & 0.007 & -0.17 & 0.06 & 0.003 & -0.06 & 0.03 & 0.062 \\
\hline Random effect (level 3) & \multicolumn{3}{|c|}{ SD } & \multicolumn{3}{|c|}{ SD } & \multicolumn{3}{|c|}{ SD } \\
\hline intercept & \multicolumn{3}{|c|}{1.97} & \multicolumn{3}{|c|}{0.71} & \multicolumn{3}{|c|}{0.91} \\
\hline
\end{tabular}




\begin{tabular}{lr|r|r} 
time & 0.06 & 0.04 & 0.01 \\
\cline { 2 - 4 } Random effect (level 2) & SD & SD & SD \\
Intercept & 12.84 & 8.47 & 4.81 \\
Time & 0.32 & 0.23 & 0.09 \\
Residual (level 1) & 7.86 & 5.42 & 3.18 \\
\hline
\end{tabular}

Note: time is measured in weeks

Table 4. Estimates from multilevel models predicting adolescent ratings of the WAI-S-T: Total score, Collaboration Subscale, and Bond Subscale.

\begin{tabular}{|c|c|c|c|c|c|c|c|c|c|}
\hline \multirow{2}{*}{$\begin{array}{l}\text { Variables } \\
\end{array}$} & \multicolumn{3}{|c|}{ WAI-S-T overall } & \multicolumn{3}{|c|}{ WAI-S-T Collaboration } & \multicolumn{3}{|c|}{ WAI-S-T Bond } \\
\hline & Estimate & $\mathbf{S E}$ & $\mathbf{p}$ & Estimate & SE & $\mathbf{p}$ & Estimate & $\mathbf{S E}$ & $\mathbf{p}$ \\
\hline \multicolumn{10}{|l|}{ Fixed effect } \\
\hline Intercept & 51.42 & 1.72 & 0.000 & 33.01 & 1.23 & 0.000 & 18.48 & 0.54 & 0.000 \\
\hline Time & 0.15 & 0.04 & 0.001 & 0.10 & 0.03 & 0.003 & 0.06 & 0.01 & 0.000 \\
\hline \multicolumn{10}{|l|}{ Arm (ref: STPP) } \\
\hline Arm: BPI & 6.37 & 2.50 & 0.013 & 4.90 & 1.80 & 0.008 & 1.29 & 0.75 & 0.913 \\
\hline Arm: CBT & 7.51 & 2.48 & 0.003 & 5.12 & 1.78 & 0.005 & 2.40 & 0.75 & 0.002 \\
\hline Random effect (level 3) & & & SD & & SD & & & SD & \\
\hline intercept & & & 5.57 & & 6.04 & & & 2.04 & \\
\hline time & & & 0.11 & & 0.19 & & & 0.05 & \\
\hline Random effect (level 2) & & & SD & & SD & & & SD & \\
\hline Intercept & & & 8.19 & & 3.79 & & & 2.04 & \\
\hline Time & & & 0.22 & & 0.05 & & & 0.05 & \\
\hline Residual (level 1) & & & 5.44 & & 4.03 & & & 1.74 & \\
\hline
\end{tabular}

Note: time is measured in weeks 
Figure 1. Observed means and model predictions of the WAI-S and WAI-S-T overall score
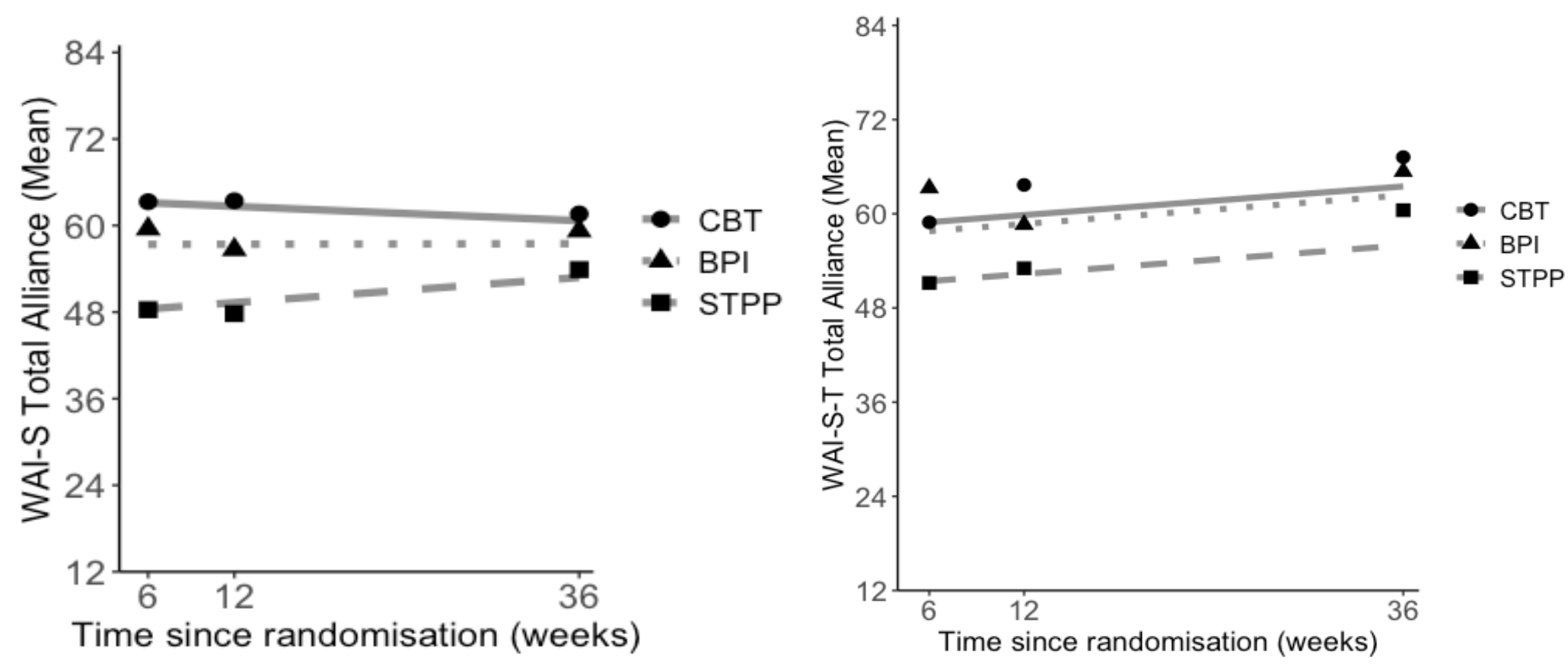